\title{
COMMUNICATION
}

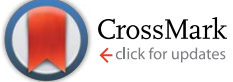

Cite this: RSC Adv., 2015, 5, 29312

Received 29th January 2015 Accepted 17th March 2015

DOI: $10.1039 / c 5 r a 01791 b$

www.rsc.org/advances

\section{Trifluoromethyl syn- or anti- $\gamma$-amino alcohols by one-pot solvent-free Mannich-type reactions under temperature control $\dagger$}

\author{
Stefania Fioravanti, ${ }^{*}$ Luca Parise, Alessia Pelagalli, Lucio Pellacani and Laura Trulli
}

Starting from trifluoroacetaldehyde ethyl hemiacetal, chiral amines and suitable aldehydes, diastereomerically pure fluorinated syn- or anti- $\gamma$-amino alcohols can be obtained by a friendly one-pot solventfree L-proline catalysed Mannich-type reaction only by changing the temperature.

\section{Introduction}

The selective organocatalytic Mannich reactions, ${ }^{\mathbf{1}}$ particularly those catalysed by proline, represent one of the most important methods for the asymmetric formation of carbon-carbon bonds, leading to optically active $\beta$-amino carbonyl compounds, versatile synthetic building blocks for the preparation of many biologically important nitrogen-containing compounds. ${ }^{2}$ Several approaches to obtain different $\beta$-amino aldehydes, precursors of $\beta$-amino acids ${ }^{3}$ or $\gamma$-amino alcohols, ${ }^{4}$ have been reported, but only a few papers have considered the synthesis of analogous fluorinated molecules. ${ }^{5}$

Proline has been reported to catalyse the addition of acetone to a few fluorinated aldimines, giving the corresponding addition compounds in generally low yields. Mostly, the reactions were successful only by using acetone as both solvent and reagent, other ketones failing under these conditions. ${ }^{6}$ Fustero and co-workers have reported a highly diastereo- and enantioselective synthesis of fluorinated $s y n$ - $\gamma$-amino alcohols by an L-proline catalysed Mannich-type reaction on fluorinated aldimines with aliphatic aldehydes. ${ }^{7}$ The reactions were carried out using $N$-methyl-2-pyrrolidone (NMP) as solvent at $-20{ }^{\circ} \mathrm{C}$, following the same reaction conditions used by Hayashi. ${ }^{8}$ In order to improve the chemical yields, the reaction conditions

Dipartimento di Chimica, Università degli Studi di Roma "La Sapienza", P.le Aldo Moro 5, I-00185 Roma, Italy. E-mail: stefania.fioravanti@uniroma1.it; Fax: +39 06490631; Tel: +390649913098

$\dagger$ Electronic supplementary information (ESI) available: General procedures, analytical and spectroscopic data, ${ }^{1} \mathrm{H}$ and ${ }^{13} \mathrm{C}$ NMR spectra of all new compounds. See DOI: 10.1039/c5ra01791b (time, solvent, temperature, amount of aldehyde and catalyst) were changed but only a temperature increase (stepwise from -20 to $0{ }^{\circ} \mathrm{C}$ ) gave significantly better yields. More recently, the same authors reported the synthesis of fluorinated anti- $\gamma$-amino alcohols by using Jørgensen-Hayashi's aryl prolinols ${ }^{9}$ as catalysts in the Mannich-type reaction. ${ }^{\mathbf{1 0}}$

For some years we were interested in the chemistry of fluorine and in particular in the synthesis and reactivity of trifluoromethyl imines to obtain trifluoromethylated nitrogencontaining compounds. ${ }^{11}$ At the same time, we continued to study the optimization of some synthetic procedures through new solvent-free and/or one-pot methodologies, ${ }^{12}$ according to the guidelines of green chemistry and the ever-increasing demand for environment respect. ${ }^{13}$ Furthermore, compared to conventional methods that may also require the use of an excess of organic solvent which then must be removed and properly disposed of in the environment, a solvent-free organic process often allows to decrease the reaction times.

Then, interested to develop a green procedure to synthesise nitrogen-containing organofluorine compounds and inspired by our recent results, a one-pot solvent-free L-proline catalysed Mannich-type reaction ${ }^{\mathbf{1 4}}$ was attempted (Scheme 1).

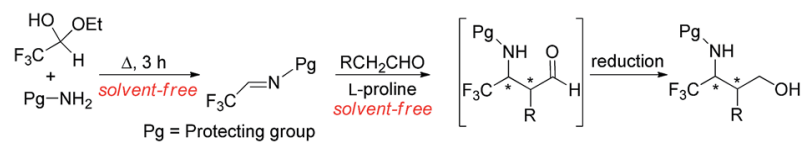

Scheme 1 One-pot solvent-free L-proline catalysed Mannich-type reaction.

\section{Results and discussion}

Commercial benzylamine (1a) or $p$-methoxyaniline (1) $)$ were chosen to test a possible influence on the addition reaction of an alkyl or aromatic residue on the nitrogen imine, especially considering that, while the benzylic group can be removed under mild conditions, the removal of the most commonly used 
$p$-methoxyphenyl (PMP) group from nitrogen requires rather drastic oxidative conditions involving harmful reagents, such as ceric ammonium nitrate, which are not compatible with a green procedure. As suitable carbonyl reaction partners, linear or branched aldehydes were considered in the solvent-free Mannichtype step. Compound $\mathbf{1 a}$ or $\mathbf{1 b}$ were added in equimolar ratio to trifluoroacetaldehyde ethyl hemiacetal heating to $120^{\circ} \mathrm{C}$ for $3 \mathrm{~h} .{ }^{11 c}$ After bringing the mixtures to room temperature, L-proline (10 mol\%) and different aldehydes were fast added. ${ }^{15}$ The solventfree Mannich-type reactions were followed by ${ }^{19} \mathrm{~F}$ NMR (1 h) and then, after cooling to $0{ }^{\circ} \mathrm{C}, \mathrm{NaBH}_{4}{ }^{2 i}$ was added to obtain trifluoromethylated syn- $\gamma$-amino alcohols 2-4a,b (Table 1).

Table 1 Solvent-free synthesis of trifluoromethylated syn- $\gamma$-amino alcohols

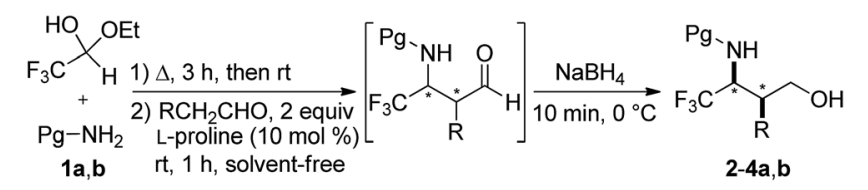

\begin{tabular}{lllll}
\hline Entry & Pg & $\mathrm{R}$ & Product & Yield $^{a}(\%)$ \\
\hline 1 & $\mathrm{Bn}$ & $i \mathrm{Pr}$ & $\mathbf{2 a}$ & 51 \\
2 & & $\mathrm{Pr}$ & $\mathbf{3 a}$ & 54 \\
3 & $\mathrm{Me}$ & $\mathbf{4 a}$ & 63 \\
& & & \\
4 & & & $\mathbf{2 b}$ & 49 \\
5 & $\mathrm{PMP}$ & $\mathrm{Pr}$ & $\mathbf{3 b}$ & 51 \\
6 & & $\mathrm{Me}$ & $\mathbf{4 b}$ & 60 \\
${ }^{a}$ After purification by flash chromatography.
\end{tabular}

No significant difference in reactivity was observed and the expected compounds were obtained in all cases in satisfactory yields as pure syn diastereomers. However, surprisingly no enantioselectivity was observed, the obtained amino alcohols 2-4a,b resulting a racemic mixture by chiral HPLC analysis. Thinking that these unexpected data could be due to the reaction temperature, we repeated the solvent-free Mannich step lowering the temperature (from 0 to $-20{ }^{\circ} \mathrm{C}$ ) but also under these conditions the enantioselectivity of the reactions did not change (see ESI $\dagger$ ) and only a decrease of the yields and an increase of the reaction times were observed. Also by raising the reaction temperature $\left(40^{\circ} \mathrm{C}\right)$, no change in stereoselectivity was observed. ${ }^{16}$

Comparing our data with the results reported in the literature for analogous addition reactions on trifluoromethyl aldimines, ${ }^{7}$ it is possible to suppose that the use of a polar solvent is crucial on the enantioselective outcome of proline catalysed Mannich-type reaction. It is well known that the enantioselectivity of this reaction is strongly controlled in the transition state by an intramolecular proton transfer from the proline carboxylic group to the nitrogen atom of imine in $E$ configuration. $^{9 b, 17}$ As a consequence, the obtained results seem to suggest, in the absence of a polar solvent (DMSO, DMF, NMP), the existence of an equilibrium between I and II (Fig. 1), despite the intramolecular hydrogen bond can be formed only in $\mathbf{I}$.
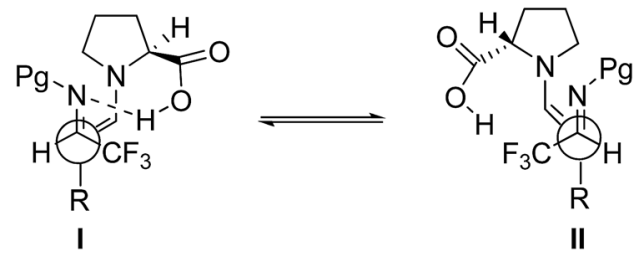

Fig. 1 Equilibrium between the two possible syn approach modes of the reaction partners.

To the best of our knowledge, in the literature only one intriguing solvent effect was reported for a direct Mannich-type reaction performed with aromatic aldehydes, anilines and cyclic ketones in the presence of $\mathrm{ZrOCl}_{2} \cdot 8 \mathrm{H}_{2} \mathrm{O}$ as catalyst. Whereas the authors observed a low stereoselectivity when the threecomponent Mannich reactions were performed in aqueous or organic solvent, a very excellent anti selectivity and yield increase were obtained working under solvent-free conditions. ${ }^{18}$

Hoping to gain more information, starting from 1a the Mannich-type reaction was repeated with isovaleraldehyde in the presence of L-proline methyl ester as catalyst, thus excluding the possibility of an intramolecular hydrogen bond on the transition state (Scheme 2).

As expected, 2a was obtained as an enantiomeric mixture of only syn isomers, in time and yields similar to those obtained in the reaction performed by using L-proline as catalyst.

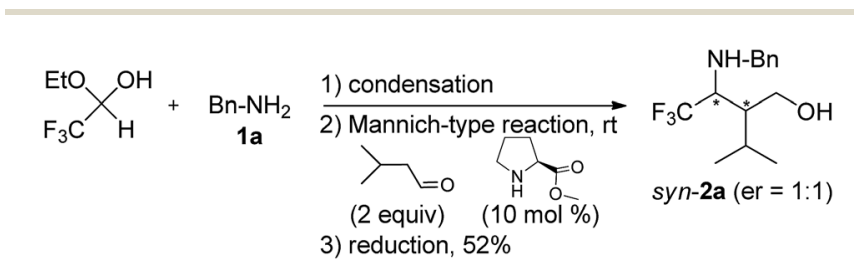

Scheme 2 L-Proline methyl ester catalysed Mannich-type reaction.

We decided to attempt a Mannich-type reaction under the green conditions, but starting from the chiral primary amines $(R)$-1-phenylethylamine (5a) and ( $R$ )-1-( $p$-methoxyphenyl) ethylamine $(5 \mathbf{b}),{ }^{19}$ in the hope that the presence of a stereocentre in the $\beta$ position to the electrophilic site of not isolated $(R, E)$ aldimines $\mathbf{6 a}, \mathbf{b}$ could influence the diastereoselective reaction outcome. So, starting from chiral materials the same solventfree one-pot procedure was repeated at different temperatures. The results are reported in Table $2 .^{20}$

While at room temperature (Table 2, entries 1, 4, 7 and 10) a syn/anti mixture was always obtained, by changing the reaction temperature only the syn isomers $\left(40^{\circ} \mathrm{C}\right.$, Table 2, entries $2,5,8$ and 11) or the anti isomers $\left(0^{\circ} \mathrm{C}\right.$, Table 2, entries 3, 6, 9 and 12) were observed, as shown by means of ${ }^{19} \mathrm{~F}$ NMR analyses performed on the crude mixtures (Fig. 2).

Only syn and/or only anti diastereomer was always formed regardless of reaction temperature, the chiral stereocentre on the benzyl residue strongly affecting the stereoselective reaction outcome. Considering the absolute configurations of the new chiral centres $(S, S$ for syn-7a,, $\mathbf{b}$ and $S, R$ for anti-7' $\mathbf{a}, \mathbf{b})$ determined 


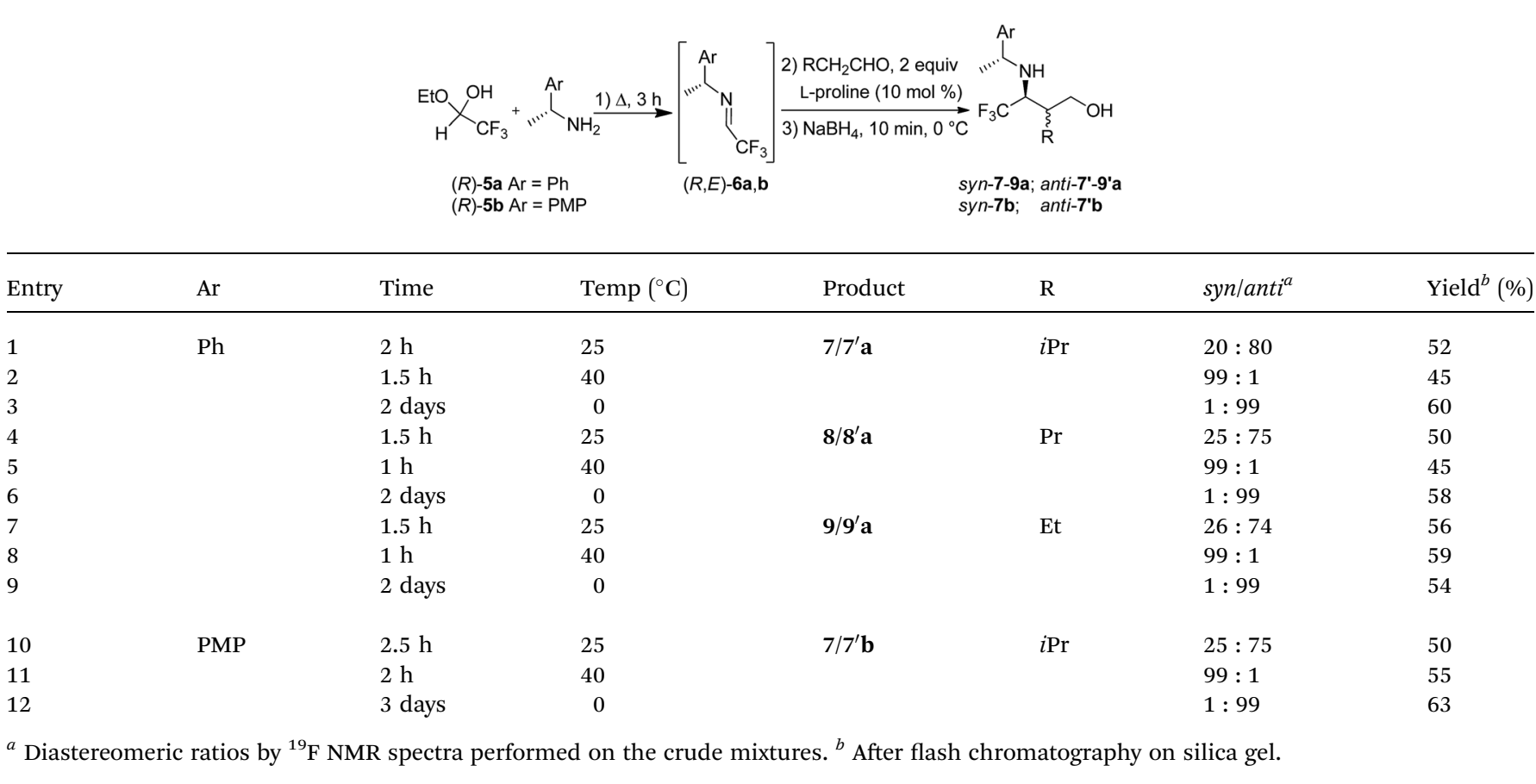

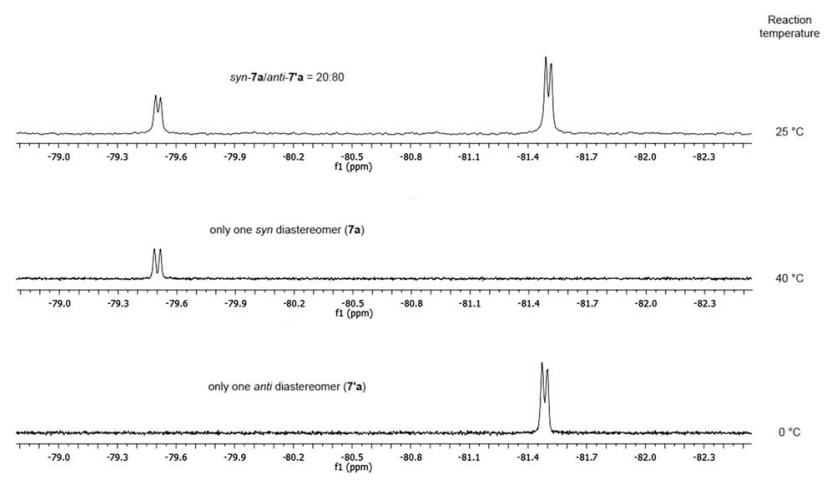

Fig. 2 Comparison of ${ }^{19} \mathrm{~F}$ NMR spectra of crude mixtures performed at different temperatures (Table 2, entries $1-3$ ).

by $2 \mathrm{D}$ NOESY ${ }^{1} \mathrm{H}$ NMR spectra and also confirmed by the chemical transformation of $\mathbf{1 0}^{\prime}$ a to a known chiral trifluoromethyl primary amine ${ }^{\mathbf{1 0}}$ (see ESI $\dagger$ ), the attack of the intermediate enamine can only take place through transition states III or IV respectively (Fig. 3), in both cases only on the $S i$ prochiral face of trifluoromethyl imines $(R, E)-\mathbf{6 a}, \mathbf{b}$.

As a first study on the role of the L-proline in the reported reactions, starting from amine $\mathbf{3 a}$, solvent-free one-pot addition reactions were repeated with isovaleraldehyde without catalyst or by using D-proline or Hayashi's catalyst $[(S)$ - $\alpha, \alpha$-diphenylprolinol] as catalyst. ${ }^{21}$ The results are reported in Table 3.

While the reactions performed without catalyst ${ }^{22}$ led to an equimolar mixture of pure diastereomers syn-7a and anti-7'a (entries 1-3), unexpectedly the use of D-proline as catalyst gave the same stereochemical results $[(S, S, R)-s y n-7 \mathbf{a}$ (entry 4$)$ and $(R, S, R)$-anti-7'a (entry 5)] already obtained by working in the

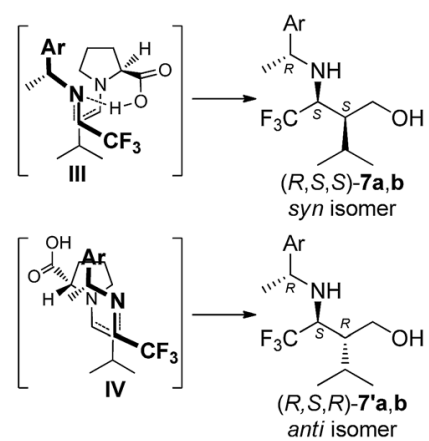

Fig. 3 Transition states for syn and anti isomers.

presence of its enantiomer L-proline (see Table 2). On the contrary, only the pure anti diastereomer $(R, S, R)-7^{\prime} \mathbf{a}$ (entries 6-8) was formed when the Mannich-type reaction was performed without solvent by using $(S)$ - $\alpha, \alpha$-diphenylprolinol, according to the data reported in the literature for this catalyst. ${ }^{\mathbf{1 0}}$

We can summarize some relevant data herein reported. Working under solvent-free conditions, the L-proline catalyst seems to be responsible only for the control of the syn selectivity. In fact, starting from achiral amines 1, only syn isomers were always obtained, but no enantioselective induction was observed, even if the reaction temperature was lowered up to $-20{ }^{\circ} \mathrm{C}$ (see Table 1). As a possible confirmation of this, the Mannich-type reactions performed without added catalysts but on enantiomerically pure imine $(R, E)$-6a led to obtain the expected products in a syn/anti ratio $=1: 1$, but each interestingly as optically pure diastereomeric $\gamma$-amino alcohols (see Table 3, entry 1-3). The latter results seem also to indicate that 
Table 3 Solvent-free additions without catalyst or with D-proline or (S)- $\alpha, \alpha$-diphenylprolinol as catalyst

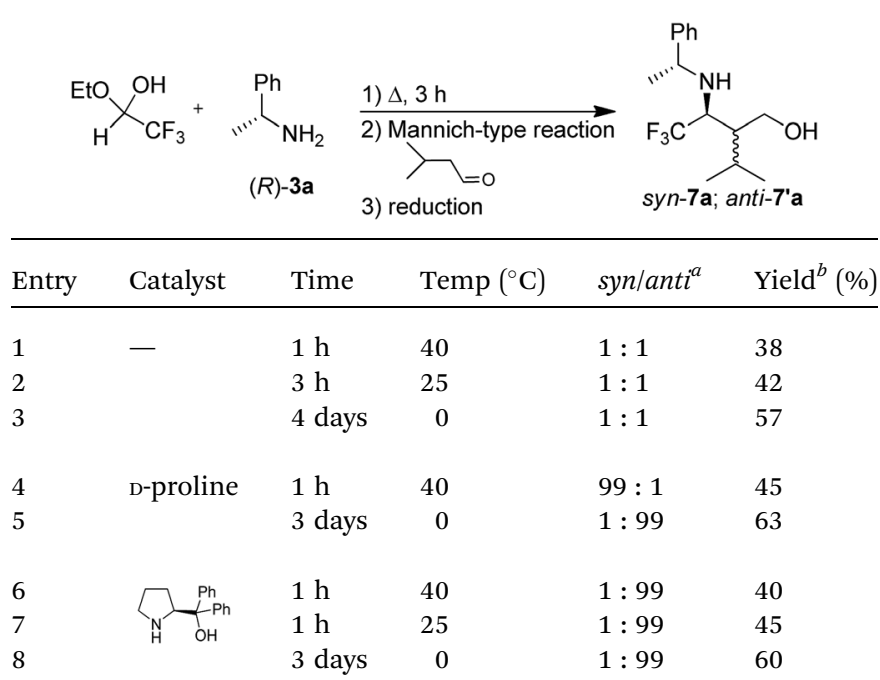

${ }^{a}$ Diastereomeric ratios by ${ }^{19} \mathrm{~F}$ NMR analysis performed on the crude mixtures. ${ }^{b}$ After flash chromatography on silica gel.

the facial stereoselective attack is controlled only by the resident stereocentre of the starting chiral amine $(R)-\mathbf{3 a}$.

An important role in the reported proline catalysed solventfree Mannich-type reactions seems to play also the reaction temperature. In fact, starting from optically pure amines, the Hayashi's catalyst leads to the optically pure anti isomers also by working at different temperatures, the steric hindrance probably controlling the reaction stereochemistry (see Table 3 , entry 6-8). On the contrary, using L-proline (Table 2) or D-proline (Table 3, entry 4,5), it is possible to obtain a complete syn or anti selective control only as a function of the reaction temperature [(40 or $0{ }^{\circ} \mathrm{C}$, respectively). This permits us to obtain diastereomerically pure $s y n$ - or anti- $\gamma$-amino alcohols working under solvent-free conditions.

\section{Conclusions}

In conclusion, a highly diastereoselective one-pot solvent-free synthesis of trifluoromethylated syn- or anti- $\gamma$-amino alcohols was reported. This efficient protocol has the advantages of environmental friendliness, good yields and operational simplicity. Unexpectedly, a strong influence of the solvent on the enantioselective outcome of the Mannich additions on trifluoromethyl aldimines was found. In fact, under solvent-free conditions only the presence of a resident stereocentre in the $\beta$ position to the imine carbon leads to the facial stereoselective control of nucleophilic attack, seeming that the added proline is able to control only the syn or the anti diastereoselectivity.

To the best of our knowledge this is the first time that the use of proline catalyst allows to obtain either syn or anti isomers just by changing the reaction temperature. Further investigations are in progress to better understand the new observed reaction outcome.

\section{Experimental section}

\section{General remarks}

IR spectra were recorded on a Perkin-Elmer 1600 FT/IR spectrophotometer in $\mathrm{CHCl}_{3}$ as solvent. ${ }^{1} \mathrm{H}$ NMR and ${ }^{13} \mathrm{C}$ NMR spectra were recorded on a VARIAN XL-300 spectrometer at 300 and $75 \mathrm{MHz}$ or on a Bruker Avance III at 400 and $101 \mathrm{MHz}$ respectively at room temperature. $\mathrm{CDCl}_{3}$ was used as solvent and $\mathrm{CHCl}_{3}$ and $\mathrm{CDCl}_{3}$ as internal standard for ${ }^{1} \mathrm{H}$ and ${ }^{13} \mathrm{C}$, respectively. ${ }^{19} \mathrm{~F}$ NMR spectra were recorded on a VARIAN XL300 spectrometer at $282.2 \mathrm{MHz}$, using $\mathrm{CDCl}_{3}$ as solvent and $\mathrm{C}_{6} \mathrm{~F}_{6}$ as internal standard. The NOESY experiments were performed with a Bruker Avance III spectrometer at $400 \mathrm{MHz}$ using $\mathrm{CDCl}_{3}$ as solvent and $\mathrm{CHCl}_{3}$ as internal standard and used to assist in structure elucidation. ${ }^{23}$ Enantiomeric ratios were determined by HPLC analyses performed with a Varian 9002 instrument equipped with a Varian $9050 \mathrm{UV} /$ Vis detector using an analytical IA Chiralcel column, HPLC grade hexane/2propanol $=95: 5$ as eluent, flow $0.9 \mathrm{~mL} \mathrm{~min}^{-1}$. HR-MS analyses were performed using a Micromass Q-TOF Micro quadrupole-time of flight (TOF) mass spectrometer equipped with an ESI source and a syringe pump. The experiments were conducted in the positive ion mode. Optical rotation was determined at $25{ }^{\circ} \mathrm{C}$ with a JASCO DIP-370 polarimetry at a wavelength of $589 \mathrm{~nm}$, using a quartz cell of $1 \mathrm{~cm}$ length.

Except for aldimine intermediate $(R, E)$-6b which is a new compound and was completely characterised, all not isolated $(E)$-trifluoromethyl aldimines are known compounds. ${ }^{11,24}$

\section{Synthesis of trifluoromethyl $\gamma$-amino alcohols. General procedure}

An equimolar solution (1 mmol) of trifluoroacetaldehyde ethyl hemiacetal and an opportune primary amine was heated to $120^{\circ} \mathrm{C}$ for $3 \mathrm{~h}\left({ }^{19} \mathrm{~F}\right.$ NMR). After bringing the reaction mixture to room temperature, s-proline $(10 \mathrm{~mol} \%)$ and an opportune aldehyde $(2 \mathrm{mmol})$ were fast added. Then, the reactions were stirred under solvent-free conditions at different temperatures $(40,25$, 0 or $\left.-20^{\circ} \mathrm{C}\right)$. When the reactions were completed $(1 \mathrm{~h}-3$ days, see Tables 1-3), anhydrous $\mathrm{Et}_{2} \mathrm{O}(3 \mathrm{~mL})$ and $\mathrm{NaBH}_{4}(2 \mathrm{mmol})$ were added at $0{ }^{\circ} \mathrm{C}$. After $10 \mathrm{~min}$ of stirring, the mixtures were quenched with a saturated aqueous $\mathrm{NH}_{4} \mathrm{Cl}$ solution, extracted with ethyl acetate and dried on $\mathrm{Na}_{2} \mathrm{SO}_{4}$. After removal of the solvents under vacuum, the expected trifluoromethyl $\gamma$-amino alcohols were obtained as pure compounds by flash chromatography on silica gel (hexane/EtOAc $=8: 2$ ).

$\left(2 R^{*}, 3 R^{*}\right)-3$-(Benzylamino)-4,4,4-trifluoro-2-isopropylbutan1-ol (syn-2a)

Yellow oil $(51 \%, 140 \mathrm{mg})$. IR: $3347 \mathrm{~cm}^{-1} .{ }^{1} \mathrm{H}$ NMR $\left(\mathrm{CDCl}_{3}\right): \delta 0.93$ $(\mathrm{d}, J=6.8 \mathrm{~Hz}, 3 \mathrm{H}), 1.02(\mathrm{~d}, J=6.8 \mathrm{~Hz}, 3 \mathrm{H}), 1.52-1.62(\mathrm{~m}, 3 \mathrm{H})$, 1.95-1.97 (m, 1H), 2.94-3.02 (m, 2H), 3.68-3.84 (m, 2H), 4.07$4.17(\mathrm{~m}, 1 \mathrm{H}), 7.26-7.37(\mathrm{~m}, 5 \mathrm{H}) .{ }^{19} \mathrm{~F}$ NMR $\left(\mathrm{CDCl}_{3}\right): \delta-80.6(\mathrm{~d}, J$ $=7.7 \mathrm{~Hz}) \cdot{ }^{13} \mathrm{C}$ NMR $\left(\mathrm{CDCl}_{3}\right): \delta 19.0,21.1,26.7,42.1,46.8,53.9$, $72.5(\mathrm{q}, J=29.3 \mathrm{~Hz}), 126.0(\mathrm{q}, J=283.6 \mathrm{~Hz}), 127.5,128.2(2 \mathrm{C})$, 128.6 (2C), 138.4. HR-MS (ESI Q-TOF) $(\mathrm{m} / \mathrm{z})[\mathrm{M}+\mathrm{H}]^{+}$calcd for $\mathrm{C}_{14} \mathrm{H}_{21} \mathrm{~F}_{3} \mathrm{NO} 276.1575$, found 276.1570 . 
$\left(2 R^{*}, 3 R^{*}\right)-4,4,4-T r i f l u o r o-2-i s o p r o p y l-3-(4-m e t h o x y p h e n y l a m i n o)-$ butan-1-ol (syn-2b)

Red oil (49\%, $142 \mathrm{mg})$. IR: $3338 \mathrm{~cm}^{-1} \cdot{ }^{1} \mathrm{H}$ NMR $\left(\mathrm{CDCl}_{3}\right): \delta 1.02$ $(\mathrm{d}, J=6.9 \mathrm{~Hz}, 3 \mathrm{H}), 1.08(\mathrm{~d}, J=6.7 \mathrm{~Hz}, 3 \mathrm{H}), 1.54(\mathrm{br}, 1 \mathrm{H}), 1.77-$ $1.79(\mathrm{~m}, 1 \mathrm{H}), 2.00-2.09(\mathrm{~m}, 1 \mathrm{H}), 3.30-3.38(\mathrm{~m}, 2 \mathrm{H}), 3.77(\mathrm{~s}, 3 \mathrm{H})$, 4.12-4.16 (m, 1H), $4.42(\mathrm{br}, 1 \mathrm{H}), 6.77-6.83(\mathrm{~m}, 4 \mathrm{H}) .{ }^{19} \mathrm{~F}$ NMR $\left(\mathrm{CDCl}_{3}\right): \delta-80.2(\mathrm{~d}, J=7.7 \mathrm{~Hz}) .{ }^{13} \mathrm{C} \mathrm{NMR}\left(\mathrm{CDCl}_{3}\right) \delta$ 19.0, 20.7, 27.5, 42.6, 44.6, 55.6, 72.0 (q, $J=29.8 \mathrm{~Hz}) 114.8(2 \mathrm{C}), 117.7(2 \mathrm{C})$, $125.8(\mathrm{q}, J=283.2 \mathrm{~Hz}), 140.6,154.4$. HR-MS (ESI Q-TOF) $(\mathrm{m} / \mathrm{z})[\mathrm{M}$ $+\mathrm{H}]^{+}$calcd for $\mathrm{C}_{14} \mathrm{H}_{21} \mathrm{~F}_{3} \mathrm{NO}_{2}$ 292.1524, found 292.1528.

$\left(2 R^{*}\right)-2-\left[\left(1 R^{*}\right)-1-(B e n z y l a m i n o)-2,2,2-\right.$ trifluoroethyl]pentan-1ol (syn-3a)

Yellow oil (54\%, $148 \mathrm{mg})$. IR: $3310 \mathrm{~cm}^{-1} .{ }^{1} \mathrm{H}$ NMR $\left(\mathrm{CDCl}_{3}\right): \delta 0.92$ $(\mathrm{t}, J=7.0 \mathrm{~Hz}, 3 \mathrm{H}), 1.34-1.47(\mathrm{~m}, 4 \mathrm{H}), 1.57-1.68(\mathrm{~m}, 2 \mathrm{H}), 1.83-$ $1.90(\mathrm{~m}, 1 \mathrm{H}), 2.87-3.09(\mathrm{~m}, 2 \mathrm{H}), 3.70-3.86(\mathrm{~m}, 2 \mathrm{H}), 3.97-4.01$ $(\mathrm{m}, 1 \mathrm{H}), 7.27-7.34(\mathrm{~m}, 5 \mathrm{H}) .{ }^{19} \mathrm{~F} \mathrm{NMR}\left(\mathrm{CDCl}_{3}\right): \delta-77.2(\mathrm{~d}, J=9.8$ $\mathrm{Hz}) .{ }^{13} \mathrm{C} \mathrm{NMR}\left(\mathrm{CDCl}_{3}\right): \delta 13.9,20.0,32.2,35.6,49.8,53.8,74.1$ (q, $J=28.0 \mathrm{~Hz}$ ), 125.8 (q, $J=283.5 \mathrm{~Hz}), 127.8(2 \mathrm{C}), 128.5,128.7$ (2C), 137.5. HR-MS (ESI Q-TOF) $(\mathrm{m} / \mathrm{z})[\mathrm{M}+\mathrm{H}]^{+}$calcd for $\mathrm{C}_{14} \mathrm{H}_{21} \mathrm{~F}_{3} \mathrm{NO}$ 276.1575 , found 276.1569 .

$\left(2 R^{*}\right)-2-\left[\left(1 R^{*}\right)-(2,2,2-\right.$ Trifluoro)-1-(4-methoxyphenylamino)ethyl]pentan-1-ol (syn-3b)

Red oil (51\%, $148 \mathrm{mg})$. IR: $3335 \mathrm{~cm}^{-1} .{ }^{1} \mathrm{H} \mathrm{NMR}\left(\mathrm{CDCl}_{3}\right): \delta 0.97(\mathrm{t}$, $J=7.1 \mathrm{~Hz}, 3 \mathrm{H}), 1.37-1.73$ (m, 5H), 1.97-2.06 (m, 1H), 3.23-3.35 (m, 2H), 3.77 (s, 3H), 3.84 (br, 1H), 4.01-4.10 (m, 1H), 6.79-6.84 $(\mathrm{m}, 4 \mathrm{H}) \cdot{ }^{19} \mathrm{~F}$ NMR $\left(\mathrm{CDCl}_{3}\right): \delta-76.9(\mathrm{~d}, J=8.5 \mathrm{~Hz}) \cdot{ }^{13} \mathrm{C} \mathrm{NMR}$ $\left(\mathrm{CDCl}_{3}\right): \delta 14.0,20.1,29.7,32.1,48.0,55.7,73.7(\mathrm{q}, J=25.9 \mathrm{~Hz})$, 114.9 (2C), 117.9 (2C), 125.8 (q, $J=283.5 \mathrm{~Hz}), 141.2$, 154.6. HRMS (ESI Q-TOF) $(m / z)[M+H]^{+}$calcd for $\mathrm{C}_{14} \mathrm{H}_{21} \mathrm{~F}_{3} \mathrm{NO}_{2}$ 292.1524, found 292.1529 .

\section{$\left(2 R^{*}, 3 R^{*}\right)-3-(B e n z y l a m i n o)-4,4,4-t r i f l u o r o-2-m e t h y l b u t a n-1-o l$} (syn-4a)

Yellow oil (54\%, $133 \mathrm{mg})$. IR: $3341 \mathrm{~cm}^{-1} .{ }^{1} \mathrm{H}$ NMR $\left(\mathrm{CDCl}_{3}\right): \delta 0.97$ $(\mathrm{d}, J=7.0 \mathrm{~Hz}, 3 \mathrm{H}), 1.60-1.75(\mathrm{~m}, 2 \mathrm{H}), 2.38-2.52(\mathrm{~m}, 1 \mathrm{H}), 3.45-$ $3.61(\mathrm{~m}, 2 \mathrm{H}), 3.68-3.84(\mathrm{~m}, 2 \mathrm{H}), 4.27-4.37$ (m, 1H), 7.26-7.40 $(\mathrm{m}, 5 \mathrm{H}) \cdot{ }^{19} \mathrm{~F}$ NMR $\left(\mathrm{CDCl}_{3}\right): \delta-79.7(\mathrm{~d}, J=7.7 \mathrm{~Hz}) \cdot{ }^{13} \mathrm{C} \mathrm{NMR}$ $\left(\mathrm{CDCl}_{3}\right): \delta 12.4,28.1,51.4,60.9(\mathrm{q}, J=29.3 \mathrm{~Hz}), 64.6,128.2(2 \mathrm{C})$, 128.4, 128.6 (2C), 128.9 (q, $J=283.7 \mathrm{~Hz}$ ), 139.4. HRMS (ESI QTOF) $(\mathrm{m} / z)[\mathrm{M}+\mathrm{H}]^{+}$calcd for $\mathrm{C}_{12} \mathrm{H}_{17} \mathrm{~F}_{3} \mathrm{NO} 248.1262$, found 248.1270 .

(R,E)-1-(4-Methoxyphenyl)- $N$-(2,2,2-trifluoroethylidene)ethanamine $(6 b)$

Colourless liquid $(95 \%, 220 \mathrm{mg}) \cdot[\alpha]_{\mathrm{D}}=-42.0(c=1 \mathrm{~g}$ per $\left.100 \mathrm{~mL}, \mathrm{CHCl}_{3}\right)$. IR: $1660 \mathrm{~cm}^{-1} .{ }^{1} \mathrm{H}$ NMR $\left(\mathrm{CDCl}_{3}\right) \delta 1.56(\mathrm{~d}, J=$ $6.7 \mathrm{~Hz}, 3 \mathrm{H}$ ), 3.81 (s, 3H), 4.58 (q, $J=6.7 \mathrm{~Hz}, 1 \mathrm{H}), 6.88-7.25$ $(\mathrm{m}, 4 \mathrm{H}), 7.59(\mathrm{q}, J=3.3 \mathrm{~Hz}, 1 \mathrm{H}) .{ }^{19} \mathrm{~F}$ NMR $\left(\mathrm{CDCl}_{3}\right): \delta-74.5(\mathrm{~d}$, $J=7.8 \mathrm{~Hz}) .{ }^{13} \mathrm{C} \mathrm{NMR}\left(\mathrm{CDCl}_{3}\right) \delta 23.6,55.2,68.0,114.1(2 \mathrm{C}), 126.1$ $(\mathrm{q}, J=273.9 \mathrm{~Hz}), 127.8(2 \mathrm{C}), 131.7,139.5,147.7(\mathrm{q}, J=38.1 \mathrm{~Hz})$. HR-MS (ESI Q-TOF) $(\mathrm{m} / \mathrm{z})[\mathrm{M}+\mathrm{H}]^{+}$calcd for $\mathrm{C}_{11} \mathrm{H}_{13} \mathrm{~F}_{3} \mathrm{NO}$ 232.0949, found 232.0941.
$(2 S, 3 S)-4,4,4$-Trifluoro-2-isopropyl-3-[(R)-1-phenylethylamino]butan-1-ol (syn-7a)

Yellow oil $(52 \%, 150 \mathrm{mg}) \cdot[\alpha]_{\mathrm{D}}=+9.0(c=1 \mathrm{~g}$ per $100 \mathrm{~mL}$, $\left.\mathrm{CHCl}_{3}\right)$. IR: $3340 \mathrm{~cm}^{-1} \cdot{ }^{1} \mathrm{H} \mathrm{NMR}\left(\mathrm{CDCl}_{3}\right): \delta 0.82(\mathrm{~d}, J=6.7 \mathrm{~Hz}$, $3 \mathrm{H}), 0.96(\mathrm{~d}, J=6.6 \mathrm{~Hz}, 3 \mathrm{H}), 1.44(\mathrm{~d}, J=6.3 \mathrm{~Hz}, 3 \mathrm{H}), 1.53-1.63$ (m, 2H), 1.95-1.97 (m, 1H), 2.27 (br, 1H), 2.73-2.86 (m, 2H), 3.77 $(\mathrm{q}, J=6.3,1 \mathrm{H}), 4.06-4.15(\mathrm{~m}, 1 \mathrm{H}), 7.26-7.36(\mathrm{~m}, 5 \mathrm{H}) .{ }^{19} \mathrm{~F} \mathrm{NMR}$ $\left(\mathrm{CDCl}_{3}\right): \delta-77.4(\mathrm{~d}, J=7.4 \mathrm{~Hz}) .{ }^{13} \mathrm{C} \mathrm{NMR}\left(\mathrm{CDCl}_{3}\right) \delta 17.9,20.1$, 22.1, 26.4, 41.1, 44.5, 58.6, 71.7 (q, $J=29.7 \mathrm{~Hz}), 127.2(2 \mathrm{C}), 128.2$ (q, $J=283.2 \mathrm{~Hz}), 128.7,129.2(2 \mathrm{C}), 138.8$. HR-MS (ESI Q-TOF) $(\mathrm{m} / \mathrm{z})[\mathrm{M}+\mathrm{H}]^{+}$calcd for $\mathrm{C}_{15} \mathrm{H}_{23} \mathrm{~F}_{3} \mathrm{NO} 290.1732$, found 290.1735 .

$(2 R, 3 S)-4,4,4$-Trifluoro-2-isopropyl-3-[(R)-1-phenylethylamino]butan-1-ol (anti-7'a)

Yellow oil $(57 \%, 144 \mathrm{mg}) \cdot[\alpha]_{\mathrm{D}}=+18.0(c=1 \mathrm{~g}$ per $100 \mathrm{~mL}$, $\left.\mathrm{CHCl}_{3}\right)$. IR: $3335 \mathrm{~cm}^{-1} .{ }^{1} \mathrm{H} \mathrm{NMR}\left(\mathrm{CDCl}_{3}\right): \delta 0.80(\mathrm{~d}, J=6.9 \mathrm{~Hz}$, $3 \mathrm{H}), 0.90(\mathrm{~d}, J=6.7 \mathrm{~Hz}, 3 \mathrm{H}), 1.26(\mathrm{br}, 1 \mathrm{H}), 1.60(\mathrm{~d}, J=6.5 \mathrm{~Hz}$, $3 \mathrm{H}), 1.77-1.83(\mathrm{~m}, 1 \mathrm{H}), 1.96-2.02(\mathrm{~m}, 1 \mathrm{H}), 2.81-2.87(\mathrm{~m}, 1 \mathrm{H})$, 3.52 (br, $1 \mathrm{H}), 4.00$ (q, $J=6.4,1 \mathrm{H}), 4.15-4.20$ (m, 1H), 7.29-7.41 $(\mathrm{m}, 5 \mathrm{H}) \cdot{ }^{19} \mathrm{~F}$ NMR $\left(\mathrm{CDCl}_{3}\right): \delta-78.8(\mathrm{~d}, J=4.9 \mathrm{~Hz}) \cdot{ }^{13} \mathrm{C} \mathrm{NMR}$ $\left(\mathrm{CDCl}_{3}\right): \delta 18.1,21.0,23.3,26.4,41.3,44.6,58.5,71.8(\mathrm{q}, J=29.7$ $\mathrm{Hz}), 125.5$ (q, $J=283.0$ ), 127.0 (2C), 128.4, 129.0 (2C), 139.8. HRMS (ESI Q-TOF) $(m / z)[M+H]^{+}$calcd for $\mathrm{C}_{15} \mathrm{H}_{23} \mathrm{~F}_{3} \mathrm{NO} 290.1732$, found 290.1727.

$(2 S, 3 S)-4,4,4-T r i f l u o r o-2-i s o p r o p y l-3-[(R)-1-(4-m e t h o x y p h e n y l)-$ ethylamino]butan-1-ol (syn-7b)

Yellow oil $(52 \%, 166 \mathrm{mg}) \cdot[\alpha]_{\mathrm{D}}=+25\left(c=1 \mathrm{~g}\right.$ per $\left.100 \mathrm{~mL}, \mathrm{CHCl}_{3}\right)$. IR: $3330 \mathrm{~cm}^{-1} .{ }^{1} \mathrm{H} \mathrm{NMR}\left(\mathrm{CDCl}_{3}\right): \delta 0.83(\mathrm{~d}, J=6.8 \mathrm{~Hz}, 3 \mathrm{H}), 0.98$ $(\mathrm{d}, J=6.7 \mathrm{~Hz}, 3 \mathrm{H}), 1.37$ (d, $J=6.6 \mathrm{~Hz}, 3 \mathrm{H}), 1.50-1.53(\mathrm{~m}, 2 \mathrm{H})$, 1.87-1.97 (m, 1H), 2.64-2.85 (m, 2H), 3.65 (q, $J=6.6,1 \mathrm{H}), 3.74$ (br, $1 \mathrm{H}), 3.80$ (s, 3H), 4.04-4.13 (m, 1H), 6.86-7.16 (m, 4H). ${ }^{19} \mathrm{~F}$ $\operatorname{NMR}\left(\mathrm{CDCl}_{3}\right): \delta-80.4(\mathrm{~d}, J=4.6 \mathrm{~Hz}) .{ }^{13} \mathrm{C} \mathrm{NMR}\left(\mathrm{CDCl}_{3}\right): \delta 18.9$, 21.2, 23.5, 26.5, 42.1, 45.0, 55.2, 57.9, 72.5 (q, $J=29.7 \mathrm{~Hz}), 114.1$ (2C), 125.8 (q, $J=283.5 \mathrm{~Hz}), 127.4(2 \mathrm{C}), 135.4,158.9$. HR-MS (ESI Q-TOF) $(\mathrm{m} / \mathrm{z})[\mathrm{M}+\mathrm{H}]^{+}$calcd for $\mathrm{C}_{16} \mathrm{H}_{25} \mathrm{~F}_{3} \mathrm{NO}_{2} 320.1837$, found 320.1843 .

$(2 R, 3 S)-4,4,4-T r i f l u o r o-2-i s o p r o p y l-3-[(R)-1-(4-m e t h o x y p h e n y l)-$ ethylamino]butan-1-ol (anti-7'b)

Yellow oil $(60 \%, 192 \mathrm{mg}) \cdot[\alpha]_{\mathrm{D}}=+15.0(c=1 \mathrm{~g}$ per $100 \mathrm{~mL}$, $\left.\mathrm{CHCl}_{3}\right)$. IR: $3350 \mathrm{~cm}^{-1} .{ }^{1} \mathrm{H} \mathrm{NMR}\left(\mathrm{CDCl}_{3}\right): \delta 0.82(\mathrm{~d}, J=6.8 \mathrm{~Hz}$, $3 \mathrm{H}), 0.96(\mathrm{~d}, J=6.7 \mathrm{~Hz}, 3 \mathrm{H}), 1.42(\mathrm{~d}, J=6.7 \mathrm{~Hz}, 3 \mathrm{H}), 1.56-1.60$ $(\mathrm{m}, 1 \mathrm{H}), 1.73(\mathrm{br}, 1 \mathrm{H}), 1.88-1.98(\mathrm{~m}, 1 \mathrm{H}), 2.69-2.85(\mathrm{~m}, 2 \mathrm{H}), 3.3$ (br, $1 \mathrm{H}), 3.74$ (q, $J=6.7,1 \mathrm{H}), 3.80(\mathrm{~s}, 3 \mathrm{H}), 4.04-4.15(\mathrm{~m}, 1 \mathrm{H})$, 6.89-721 (m, 4H). ${ }^{19} \mathrm{~F} \mathrm{NMR}\left(\mathrm{CDCl}_{3}\right): \delta-77.1(\mathrm{~d}, J=7.4 \mathrm{~Hz}) \cdot{ }^{13} \mathrm{C}$ NMR $\left(\mathrm{CDCl}_{3}\right): \delta 18.7,21.1,23.2,26.5,41.9,44.9,52.2,57.9,72.4$ (q, $J=29.3 \mathrm{~Hz}$ ), 114.2 (2C), 125.9 (q, $J=283.5 \mathrm{~Hz}), 127.5$ (2C), 134.6, 159.1. HR-MS (ESI Q-TOF) $(\mathrm{m} / \mathrm{z})[\mathrm{M}+\mathrm{H}]^{+}$calcd for $\mathrm{C}_{16} \mathrm{H}_{25} \mathrm{~F}_{3} \mathrm{NO}_{2}$ 320.1837, found 320.1842.

(2S)-2-[(1S)-2,2,2-Trifluoro-1-\{[(1R)-1-phenylethyl]amino\}ethyl]pentan-1-ol (syn-8a)

Yellow liquid $(45 \%, 130 \mathrm{mg}) \cdot[\alpha]_{\mathrm{D}}=+12.0(c=1 \mathrm{~g}$ per $100 \mathrm{~mL}$, $\left.\mathrm{CHCl}_{3}\right)$. IR: $3308 \mathrm{~cm}^{-1} \cdot{ }^{1} \mathrm{H}$ NMR $\left(\mathrm{CDCl}_{3}\right): \delta 0.88(\mathrm{t}, J=7.3 \mathrm{~Hz}$, 
$3 \mathrm{H}), 1.19-1.42(\mathrm{~m}, 5 \mathrm{H}), 1.49(\mathrm{~d}, J=6.7 \mathrm{~Hz}, 3 \mathrm{H}), 1.53-1.60(\mathrm{~m}$, 1H), 1.84-1.91 (m, 1H), 2.65-2.96 (m, 2H), $3.83(\mathrm{q}, 6.7 \mathrm{~Hz}, 1 \mathrm{H})$, 3.95-4.02 (m, 1H), 7.29-7.39 (m, 5H). ${ }^{19} \mathrm{~F} \mathrm{NMR}\left(\mathrm{CDCl}_{3}\right): \delta-77.2$ $(\mathrm{d}, J=7.8 \mathrm{~Hz}) .{ }^{13} \mathrm{C} \mathrm{NMR}\left(\mathrm{CDCl}_{3}\right): \delta 13.9,19.9,22.9,31.8,35.4$, 48.1, 58.7, 73.8 (q, $J=29.4 \mathrm{~Hz}$ ), 125.7 (q, $J=283.5 \mathrm{~Hz}$ ), 126.6 (2C), 127.9, 128.9 (2C), 141.6. HR-MS (ESI Q-TOF) $(\mathrm{m} / \mathrm{z})[\mathrm{M}+\mathrm{H}]^{+}$ calcd for $\mathrm{C}_{15} \mathrm{H}_{23} \mathrm{~F}_{3} \mathrm{NO} 290.1732$, found 290.1741.

(2R)-2-[(1S)-2,2,2-Trifluoro-1- $\{[(1 R)-1$-phenylethyl $]$ amino $\}-$ ethyl]pentan-1-ol (anti-8' a)

Yellow liquid $(58 \%, 168 \mathrm{mg}) \cdot[\alpha]_{\mathrm{D}}=+24.0(c=1 \mathrm{~g}$ per $100 \mathrm{~mL}$, $\left.\mathrm{CHCl}_{3}\right)$. IR: $3312 \mathrm{~cm}^{-1} \cdot{ }^{1} \mathrm{H} \mathrm{NMR}\left(\mathrm{CDCl}_{3} \delta 0.85(\mathrm{t}, J=7.3 \mathrm{~Hz}, 3 \mathrm{H})\right.$, $1.15-1.28(\mathrm{~m}, 4 \mathrm{H}), 1.31-1.46(\mathrm{~m}, 2 \mathrm{H}), 1.61(\mathrm{~d}, J=6.7 \mathrm{~Hz}, 3 \mathrm{H})$, 1.93-2.02 (m, 1H), 2.72-2.97 (m, 2H), 3.96-4.07 (m, 2H), 7.29$7.41(\mathrm{~m}, 5 \mathrm{H}) .{ }^{19} \mathrm{~F} \mathrm{NMR}\left(\mathrm{CDCl}_{3}\right): \delta-76.5(\mathrm{~d}, J=7.6 \mathrm{~Hz}) .{ }^{13} \mathrm{C} \mathrm{NMR}$ $\left(\mathrm{CDCl}_{3}\right): \delta 13.8,19.8,22.2,31.5,35.4,47.7,58.9,73.2(\mathrm{q}, J=29.5$ $\mathrm{Hz}), 127.0$ (2C), 128.3 (q, $J=281.5 \mathrm{~Hz}$ ), 128.5, 129.1 (2C), 139.3. HR-MS (ESI Q-TOF) $(\mathrm{m} / \mathrm{z})[\mathrm{M}+\mathrm{H}]^{+}$calcd for $\mathrm{C}_{15} \mathrm{H}_{23} \mathrm{~F}_{3} \mathrm{NO}$ 290.1732 , found 290.1725 .

(2S,3S)-2-Ethyl-4,4,4-trifluoro-3-\{[(1R)-1-phenylethyl $]$ amino $\}$ butan-1-ol (syn-9a)

Yellow liquid $(59 \%, 126 \mathrm{mg}) \cdot[\alpha]_{\mathrm{D}}=+12.0(c=1 \mathrm{~g}$ per $100 \mathrm{~mL}$, $\left.\mathrm{CHCl}_{3}\right)$. IR: $3335 \mathrm{~cm}^{-1} .{ }^{1} \mathrm{H}$ NMR $\left(\mathrm{CDCl}_{3}\right): \delta 0.89-0.93(\mathrm{~m}, 3 \mathrm{H})$, $1.22(\mathrm{~d}, J=6.4 \mathrm{~Hz}, 3 \mathrm{H}), 1.38-1.45(\mathrm{~m}, 2 \mathrm{H}), 1.58-1.63(\mathrm{~m}, 1 \mathrm{H})$, 2.01-2.09 (m, 1H), 2.97-3.09 (m, 3H), $3.70(\mathrm{q}, J=6.6,1 \mathrm{H}), 3.81$ $(\mathrm{q}, J=6.6 \mathrm{~Hz}, 1 \mathrm{H}), 7.21-7.37(\mathrm{~m}, 5 \mathrm{H}) .{ }^{19} \mathrm{~F} \mathrm{NMR}\left(\mathrm{CDCl}_{3}\right): \delta-77.0$ $(\mathrm{d}, J=8.1 \mathrm{~Hz}) .{ }^{13} \mathrm{C} \mathrm{NMR}\left(\mathrm{CDCl}_{3}\right): \delta 14.8,21.8,22.3,30.0,57.3$, 67.2, $74.4(\mathrm{q}, J=29.3 \mathrm{~Hz}), 126.2,126.9$ (2C), 127.0 (q, $J=279.4$ $\mathrm{Hz}), 128.4$ (2C), 143.7. HR-MS (ESI Q-TOF) $(\mathrm{m} / \mathrm{z})[\mathrm{M}+\mathrm{H}]^{+}$calcd for $\mathrm{C}_{14} \mathrm{H}_{21} \mathrm{~F}_{3} \mathrm{NO} 276.1575$, found 276.1579.

\section{$(2 R, 3 S)-2-E t h y l-4,4,4$-trifluoro-3-[(1R)-1-phenylethylamino]- butan-1-ol (anti-9'a)}

Yellow liquid $(54 \%, 148 \mathrm{mg}) \cdot[\alpha]_{\mathrm{D}}=+18.0(c=1 \mathrm{~g}$ per $100 \mathrm{~mL}$, $\left.\mathrm{CHCl}_{3}\right)$. IR: $3328 \mathrm{~cm}^{-1} \cdot{ }^{1} \mathrm{H}$ NMR $\left(\mathrm{CDCl}_{3}\right): \delta 0.89(\mathrm{t}, J=7.4 \mathrm{~Hz}$, $3 \mathrm{H}), 1.44-1.73(\mathrm{~m}, 4 \mathrm{H}), 1.57(\mathrm{~d}, J=6.6 \mathrm{~Hz}, 3 \mathrm{H}), 1.79-1.88(\mathrm{~m}$, 1H), 2.71-2.96 (m, 2H), 3.90-4.06 (m, 2H). 7.30-7.39 (m, 5H). ${ }^{19} \mathrm{~F}$ $\operatorname{NMR}\left(\mathrm{CDCl}_{3}\right): \delta-77.2(\mathrm{~d}, J=7.5 \mathrm{~Hz}) .{ }^{13} \mathrm{C} \mathrm{NMR}\left(\mathrm{CDCl}_{3}\right): \delta 11.3$, 22.8, 29.7, 37.3 , 47.7, 58.8, 73.4 (q, $J=29.0 \mathrm{~Hz}$ ), 126.6 (2C), 125.5 (q, $J=285.3 \mathrm{~Hz}), 128.0,129.0(2 \mathrm{C}), 141.7$. HR-MS (ESI Q-TOF) $(\mathrm{m} / \mathrm{z})[\mathrm{M}+\mathrm{H}]^{+}$calcd for $\mathrm{C}_{14} \mathrm{H}_{21} \mathrm{~F}_{3} \mathrm{NO} 276.1575$, found 276.1580 .

\section{Acknowledgements}

We are grateful for financial support from the Università degli Studi di Roma "La Sapienza" and the Dipartimento di Chimica of the same university.

\section{Notes and references}

1 For reviews, see: (a) M. Benohoud and Y. Hayashi, Enamine Catalysis of Mannich Reactions, in Science of Synthesis, Asymmetric Organocatalysis 1; Lewis Base and Acid Catalysts, ed. B. List, Georg Thieme Verlag KG, Stuttgart, 2012, p. 73; (b) A. Ting and S. E. Schaus, Eur. J. Org. Chem., 2007, 5797.
2 (a) S. Kobayashi, Y. Mori, J. S. Fossey and M. M. Salter, Chem. Rev., 2011, 111, 2626; (b) S. Fustero, J. F. Sanz-Cervera, J. L. Acena and M. Sanchez-Rosello, Synlett, 2009, 525; (c) R. G. Arrays and J. C. Carretero, Chem. Soc. Rev., 2009, 38, 1940; (d) J. M. M. Verkade, L. J. C. van Hemert, P. J. L. M. Quaedflieg and F. P. J. T. Rutjes, Chem. Soc. Rev., 2008, 37, 29; (e) S. Mukherjee, J. W. Yang, S. Hoffmann and B. List, Chem. Rev., 2007, 107, 5471; (f) A. Ting and S. E. Schaus, Eur. J. Org. Chem., 2007, 5797; $(g)$ G. K. Friestad and A. K. Mathies, Tetrahedron, 2007, 63, 2541; (h) A. Córdova, Acc. Chem. Res., 2004, 37, 102; (i) W. Notz, F. Tanaka, S. Watanabe, N. S. Chowdari, J. M. Turner, R. Thayumanavan and C. F. Barbas III, J. Org. Chem., 2003, 68, 9624; (j) A. Córdova, W. Notz, G. Zhong, J. M. Betancort and C. F. Barbas III, J. Am. Chem. Soc., 2002, 124, 1842.

3 (a) P. S. Bhadury, S. Yang and B.-A. Song, Curr. Org. Synth., 2012, 9, 695; (b) L. Bernardi, A. Ricci and M. ComesFranchini, Curr. Org. Chem., 2011, 15, 2210; (c) B. Weiner, W. Szymanski, D. B. Janssen, A. J. Minnaard and B. L. Feringa, Chem. Soc. Rev., 2010, 39, 1656; (d) R. G. Arrayas and J. C. Carretero, Chem. Soc. Rev., 2009, 38, 1940; (e) D. Enders, C. Wang and J. X. Liebich, Chem.-Eur. J., 2009, 15, 11058.

4 (a) Y. Chi, E. P. English, W. C. Pomerantz, W. S. Horne, L. A. Joyce, L. R. Alexander, W. S. Fleming, E. A. Hopkins and S. H. Gellman, J. Am. Chem. Soc., 2007, 129, 6050; (b) A. Ting and S. E. Schaus, Eur. J. Org. Chem., 2007, 35, 5797.

5 (a) J. L. Acena, A. E. Sorochinsky and V. A. Soloshonok, Synthesis, 2012, 44, 1591; (b) K. Mikami, S. Fustero, M. Sanchez-Rosello, J. L. Acena, V. Soloshonok and A. Sorochinsky, Synthesis, 2011, 3045; (c) X.-L. Qiu and F.-L. Qing, Eur. J. Org. Chem., 2011, 2011, 3261; (d) G. Mloston, E. Obijalska and H. Heimgartner, J. Fluorine Chem., 2010, 131, 829; (e) A. E. Sorochinsky and V. A. Soloshonok, J. Fluorine Chem., 2010, 131, 127.

6 K. Funabiki, M. Nagamori, S. Goushi and M. Matsui, Chem. Commun., 2004, 1928.

7 S. Fustero, D. Jimenez, J. F. Sanz-Cervera, M. SanchezRosello, E. Esteban and A. Simon-Fuentes, Org. Lett., 2005, 7, 3433.

8 Y. Hayashi, W. Tsuboi, I. Ashimine, T. Urushihima, M. Shoji and K. Sakai, Angew. Chem., Int. Ed., 2003, 42, 3677.

9 (a) A. Mielgo and C. Palomo, Chem. Asian J., 2008, 3, 922; (b) J. Franzen, M. Marigo, D. Fielenbach, T. C. Wabnitz, A. Kjærsgaard and K. A. Jørgensen, J. Am. Chem. Soc., 2005, 127, 18296.

10 S. Fustero, F. Mojarrad, M. D. P. Carrion, J. F. Sanz-Cervera and J. L. Acena, Eur. J. Org. Chem., 2009, 5208.

11 (a) S. Fioravanti, L. Pellacani and M. C. Vergari, Org. Biomol. Chem., 2012, 10, 8207; (b) L. Carroccia, S. Fioravanti, L. Pellacani, C. Sadun and P. A. Tardella, Tetrahedron, 2011, 67, 5375; (c) L. Carroccia, S. Fioravanti, L. Pellacani and P. A. Tardella, Synthesis, 2010, 4096.

12 (a) S. Fioravanti, L. Pellacani and M. C. Vergari, Org. Biomol. Chem., 2012, 10, 524; (b) S. Fioravanti, S. Morea, A. Morreale, L. Pellacani and P. A. Tardella, Tetrahedron, 
2009, 65, 484; (c) S. Fioravanti, L. Pellacani, P. A. Tardella and M. C. Vergari, Org. Lett., 2008, 10, 1449; (d) S. Fioravanti, L. Pellacani, S. Stabile, P. A. Tardella and R. Ballini, Tetrahedron, 1998, 54, 6169.

13 (a) K. Shanab, C. Neudorfer, E. Schirmer and H. Spreitzer, Curr. Org. Chem., 2013, 17, 1179; (b) B. C. Ranu and K. Chattopadhyay, Green Procedures for the Synthesis of Useful Molecules Avoiding Hazardous Solvents and Toxic Catalysts, in Eco-Friendly Synthesis of Fine Chemicals, ed. R. Ballini, The Royal Society of Chemistry, Cambridge, U. K., 2009, ch. 5, p. 186.

14 A. Kumar, M. K. Gupta and M. Kumar, Green Chem., 2012, 14, 290, For a review on solvent-free MCR's, see: M. S. Singh and S. Chowdhury, RSC Adv., 2012, 2, 4547.

15 First we considered the greenest possible conditions, attempting the addition reaction at room temperature, and the stability of the imine derived from $1 \mathbf{a}$ in the presence of the L-proline but no significant changes were observed by ${ }^{1} \mathrm{H}$ NMR analyses after $24 \mathrm{~h}$ of stirring at room temperature.

16 The crude mixtures appear to be a single phase even at $40^{\circ} \mathrm{C}$, the aldehyde excess behaving as a solvent.

17 (a) P. H.-Y. Cheong and K. N. Houk, J. Am. Chem. Soc., 2004, 126, 13912; (b) C. Allemann, R. Gordillo, F. R. Clemente, P. H.-Y. Cheong and K. N. Houk, Acc. Chem. Res., 2004, 37, 558; (c) A. Córdova, Chem.-Eur. J., 2004, 10, 1987; (d) S. Bahmanyar and K. N. Houk, Org. Lett., 2003, 5, 1249; (e) A. Córdova, W. Notz and C. F. Barbas III, J. Org. Chem., 2002, 67, 301; (f) B. List, R. A. Lerner and C. F. Barbas III, J. Am. Chem. Soc., 2000, 122, 2395.
18 B. Eftekhari-Sis, A. Abdollahifar, M. M. Hashemi and M. Zirak, Eur. J. Org. Chem., 2006, 5152.

19 The chiral amines carry on the nitrogen atom a benzyl residue which can be easily removed under mild hydrogenolytic conditions. In addition, the amine $\mathbf{3 a}$ is cheap. For a recent Mannich-type reaction on chiral aldimines, see: G. Callebaut, F. Colpaert, M. Nonn, L. Kiss, R. Sillanpää, K. W. Törnroos, F. Fülöp, N. De Kimpe and S. Mangelinckx, Org. Biomol. Chem., 2014, 12, 3393.

20 Starting from 5a, the Mannich-type reaction was tested even at $60{ }^{\circ} \mathrm{C}$, but only a complex crude mixture in which maybe the expected product was present in trace was obtained.

21 (a) S. Meninno and A. Lattanzi, Chem. Commun., 2013, 49, 3821; (b) Y. Hayashi, T. Itoh, S. Aratake and H. Ishikawa, Angew. Chem., Int. Ed., 2008, 47, 2082; (c) C. Palomo and A. Mielgo, Angew. Chem., Int. Ed., 2006, 45, 7876; (d) I. Ibrahem and A. Córdova, Chem. Commun., 2006, 1760; (e) Y. Chi and S. H. Gellman, J. Am. Chem. Soc., 2006, 128, 6804. 22 We can suppose that in the reactions performed without catalyst the imine intermediates act as both bases and electrophiles.

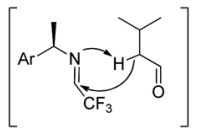

23 T. D. W. Claridge, High-Resolution NMR Techniques in Organic Chemistry, Elsevier, Amsterdam, 2nd edn, 2009.

24 H. Mimura, K. Kawada, T. Yamashita, T. Sakamoto and Y. Kikugawa, J. Fluorine Chem., 2010, 131, 477. 